\title{
Spectral and Imaging Characterization of Tabletop X-ray Lasers
}

J. Dunn, A. Ya. Faenov, T. A. Pikuz, A. L. Osterheld, S. J. Moon, K. B. Fournier, J. Nilsen, I. Yu. Skobelev, A. I. Magunov, and V. N. Shlyaptsev

This article was submitted to

7th International Conference on X-Ray Lasers, Saint-Malo, France

June 18-23, 2000

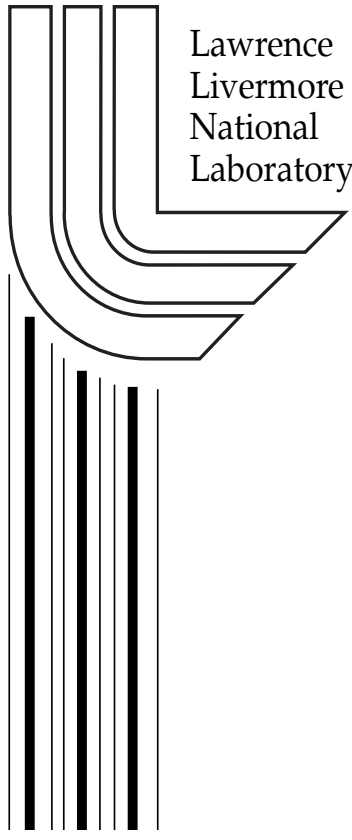

\section{December 1, 2000}




\section{DISCLAIMER}

This document was prepared as an account of work sponsored by an agency of the United States Government. Neither the United States Government nor the University of California nor any of their employees, makes any warranty, express or implied, or assumes any legal liability or responsibility for the accuracy, completeness, or usefulness of any information, apparatus, product, or process disclosed, or represents that its use would not infringe privately owned rights. Reference herein to any specific commercial product, process, or service by trade name, trademark, manufacturer, or otherwise, does not necessarily constitute or imply its endorsement, recommendation, or favoring by the United States Government or the University of California. The views and opinions of authors expressed herein do not necessarily state or reflect those of the United States Government or the University of California, and shall not be used for advertising or product endorsement purposes.

This is a preprint of a paper intended for publication in a journal or proceedings. Since changes may be made before publication, this preprint is made available with the understanding that it will not be cited or reproduced without the permission of the author.

This report has been reproduced directly from the best available copy.

Available to DOE and DOE contractors from the Office of Scientific and Technical Information

P.O. Box 62, Oak Ridge, TN 37831

Prices available from (423) 576-8401

http:/ / apollo.osti.gov/bridge/

Available to the public from the National Technical Information Service

U.S. Department of Commerce 5285 Port Royal Rd., Springfield, VA 22161

http://www.ntis.gov/

OR

Lawrence Livermore National Laboratory

Technical Information Department's Digital Library

http://www.llnl.gov/tid/Library.html 


\title{
Spectral and Imaging Characterization of Tabletop X-ray Lasers
}

\author{
J. Dunn, A. Ya. Faenov ${ }^{1}$, T. A. Pikuz ${ }^{1}$, A. L. Osterheld, S. J. Moon, K. B. Fournier, \\ J. Nilsen, I. Yu. Skobelev ${ }^{1}$, A.I. Magunov ${ }^{1}$, and V. N. Shlyaptsev ${ }^{2}$ \\ Lawrence Livermore National Laboratory, Livermore, CA 94550, USA \\ ${ }^{1}$ MISDC of VNIIFTRI, Mendeleevo, Moscow Region, 141570, Russia \\ 2 DAS, University of California Davis-Livermore, Livermore, CA 94550,USA
}

\begin{abstract}
We have performed L-shell spectroscopy and one-dimensional (1-D) imaging of a line focus plasma from a laser-heated $\mathrm{Fe}$ polished slab using the tabletop COMET laser system at the Lawrence Livermore National Laboratory. These plasmas are used to generate a Ne-like Fe transient gain $\mathrm{x}$-ray laser that is recorded simultaneously. A spherically-curved crystal spectrometer gives high resolution x-ray spectra of the $n=3-2$ and $n=4-2$ resonance lines with 1-D spatial resolution along the line focus. Spectra are presented for different laser pulse conditions. In addition, a variety of $\mathrm{x}$-ray imaging techniques are described. We discuss imaging results from a double-slit x-ray camera with a spherically-curved crystal spectrometer. We show a high resolution Fe K- $\alpha$ spectrum from the $\mathrm{x}$-ray laser target that indicates the presence of hot electrons in the $\mathrm{x}$-ray laser plasma.
\end{abstract}

\section{INTRODUCTION}

The concept of the transient collisional x-ray laser has allowed rapid development in the last 3 years leading to a dramatic $1-2$ orders of magnitude reduction in the laser driver energy to generate the population inversion [1 - 4]. This improvement is required for producing present and future laserdriven tabletop x-ray lasers. Further understanding of the transient collisional x-ray laser parameters is necessary and so performing detailed theoretical and experimental investigation of the plasma parameters is essential. In particular, the ionization, population inversion and collisional redistribution of the $\mathrm{Ne}$ - or $\mathrm{Ni}$ - like ion excited levels before and during the x-ray laser generation are important. Better understanding of the time and space irradiation nonuniformity and the influence on the overall $x$-ray laser intensity requires the study of the local distribution and dynamics along the lasing gain medium at the wavelengths relevant to the lasing transitions. This means that it is necessary to measure spatial and time distribution of line intensities of Ne-like $n=4,3-2$ resonance lines, for example, which lie in the spectral range below $20 \AA$ for mid- $Z$ materials.

In previous experimental investigations $\mathrm{x}$-ray spectra of different Ne-like ions, involving for example space- [5] or space- and time-resolution [6], were measured in x-ray laser plasmas. The modest laser energies $5-10 \mathrm{~J}$ available for tabletop, transient collisional $\mathrm{x}$-ray lasers now requires a different approach to the characterization of the x-ray laser plasma gain region where high efficiency spectrometers are essential. Secondly, good spatial resolution in 1-dimension (1-D) or 2-dimensions (2-D) is important in studying the effects of spatial non-uniformity in the plasma gain medium. High spectral resolution is another very important property for investigation of transient collisional x-ray laser medium. Ideally, the experimental instrumental resolving power should be higher than $\lambda \delta \lambda>$ 2000 to sufficiently resolve and determine the resonance line intensity of $\mathrm{Ne}$ - or Ni-like ions and their satellites. Therefore, simultaneously with x-ray laser generation, it is necessary to use much more sensitive spectrometers to make measurements of space- and time-resolved $\mathrm{x}$-ray spectra.

In this work, we briefly present results of spectrally resolved x-ray imaging of $\mathrm{Ne}$-like $\mathrm{Fe}$ transient collisional $\mathrm{x}$-ray laser gain media while simultaneously measuring the $\mathrm{x}$-ray laser output. The spectral x-ray imaging is achieved with Focusing Spectrometers with Spatial Resolution (FSSR- 
1D, -2D) instruments that satisfy most of the above requirements. We also show that by observing the Fe K- $\alpha$ spectrum from the $\mathrm{x}$-ray laser plasma medium indicates laser energy being absorbed and generating superthermal electrons.

\section{EXPERIMENTAL DESCRIPTION AND X-RAY MEASUREMENTS}

The Ne-like Fe experiments were performed on the COMET laser at LLNL. For a more complete description of the laser system and further experimental details see [7, 8]. Laser parameters were chosen for irradiating flat slab Fe targets of length up to $1 \mathrm{~cm}$ : Energy of $\sim 5 \mathrm{~J}$, of $1054 \mathrm{~nm}$ wavelength light in a 600 ps pulse was followed by a delay of 1.4 ns before utilizing a 1.2 ps excitation pulse with 5-6 J. The long pulse line focus was defocused while the short pulse was tightly focused. This gave the intensity for the plasma formation beam and short pulse excitation beam of $7 \times 10^{11} \mathrm{~W} \mathrm{~cm}^{-2}$ and $5 \times 10^{14} \mathrm{~W} \mathrm{~cm}^{-2}$ on target, respectively. The reflection echelon traveling wave excitation was used in this study. The overlap of the line foci from two segments of the reflection echelon created a hot spot in the center of the laser line focus. It was decided to leave this uncorrected in order to create a distinct spatial feature to be studied by the various slit and spectral imaging diagnostics.

Our usual x-ray diagnostics included an on-axis 1200 line $\mathrm{mm}^{-1}$ variable-spaced flat-field grating spectrometer with a back-thinned $1024 \times 1024$ charge-coupled device (CCD) and CCD x-ray slit camera with $25 \mu \mathrm{m}$ spatial resolution. These monitored the x-ray laser intensity and the line focus plasma uniformity, respectively. In addition, several FSSR spectrometers using Kodak DEF film and CCD detector arrays were employed to study the Ne-like Fe resonance line emission. These spectrometers $[9,10]$ use a spherically curved Mica crystal Bragg analyzer as the dispersive element and have numerous advantages including: (1) very high signal throughput due to the focusing properties of spherically bent crystals; (2) both high spectral and spatial resolution of a large $1 \mathrm{~cm}$ long plasma without the use of any slit or aperture; (3) a large field of view obtained simultaneously with good spatial and spectral resolution.

(a)

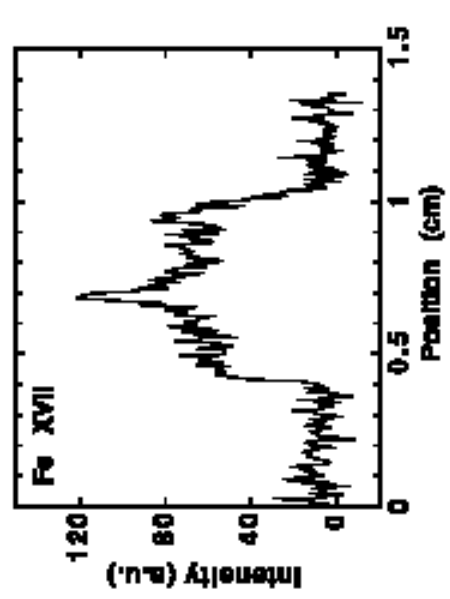

(b)

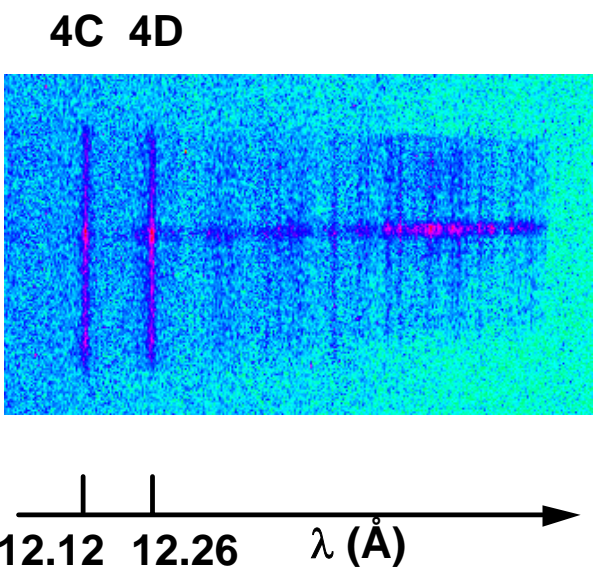

(c)

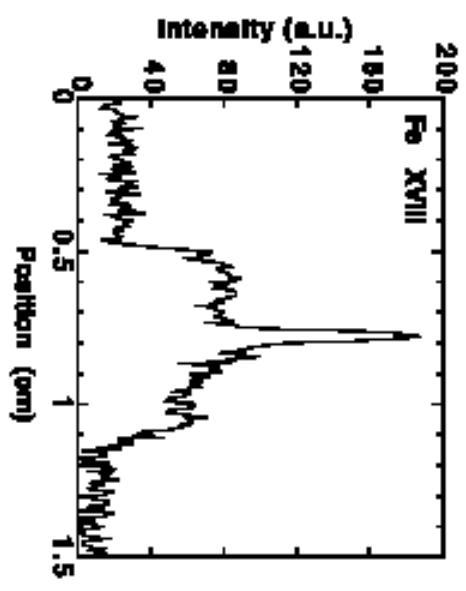

Figure 1Image and lineouts from FSSR instrument showing Ne-like Fe XVII $n=4-2$ and F-like $n=3-2$ transitions emitted from flat, $0.6 \mathrm{~cm}$ long Fe target heated by the two laser pulse configuration: subnanosecond (600 ps, $4 \mathrm{~J}$ ) followed by picosecond (1.2 ps, $4.8 \mathrm{~J}$ ) pulse with delay between pulses of $\Delta \tau=1.4 \mathrm{~ns}$. (a) Trace of 4C Ne-like Fe XVII spectral line along X-ray laser medium. (b) Spatially resolved x-ray spectral image. Wavelength dispersion direction is left - right. Spatial resolution along the line focus is in the perpendiculsr direction. (c) Trace of F-like Fe XVIII $3 p-2$ s spectral line at $12.8 \AA$ along X-ray laser medium.

This work was performed under the auspices of the U.S. Department of Energy by the University of California, Lawrence Livermore National Laboratory under contract No. W7405-Eng-48. 


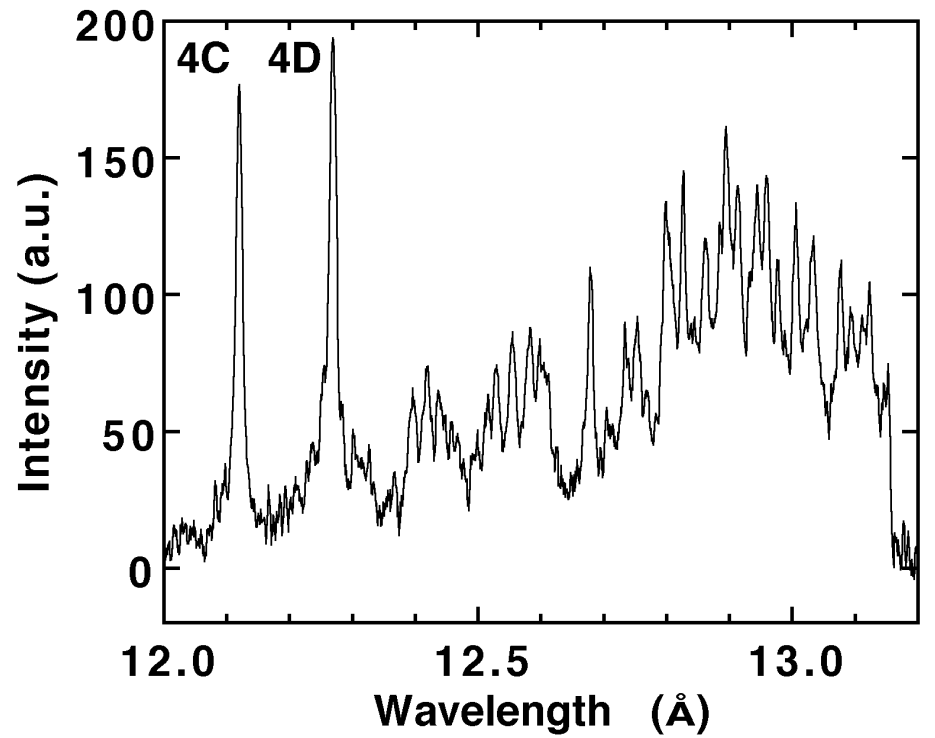

Figure 2 FSSR spectrum showing Ne-like Fe XVII $n=4-2$ and F-like, O-like $n=3-2$ transitions emitted from flat iron target under conditions described in Fig. 1. Spatially resolved x-ray spectral image with lineout through dispersion axis on center hot spot.
By firing only the 600 ps laser pulse, the pre-formed plasma ionization conditions can be studied before the short pulse heating. A strong Ne-like 3 -2 resonance signal was observed on these shots that confirmed that the $\mathrm{Ne}$ like ion stage was being generated with the first laser pulse [8]. This is contrast to the Ne-like Ge $\mathrm{x}$-ray laser experiments described by Warwick et al. where no Ne-like resonance lines were observed with the long pulse only irradiation [4]. Some comparisons between the $\mathrm{x}$-ray slit camera imaging and the FSSR spectrometer gave good agreement showing similar characteristics in intensity along the line focus for laser conditions when either of the two beams or both beams were fired. In the latter case, where the short pulse laser heated the line focus, the strongest $\mathrm{x}$-ray emission was

observed. X-ray laser output was only observed when both laser beams were fired. The line focus hot spot, mentioned earlier, showed modest enhancement of the Ne-like $3 d-2 p$ resonance line intensity. Fig.1(b) is a spectral image from the FSSR 1-D instrument recorded on film showing the Ne-like Fe $4-2$ resonance lines and the F-like Fe $3-2$ lines between 12 and $13 \AA$ with spatial resolution along the line focus of a $0.6 \mathrm{~cm}$ target. Both laser pulses were incident on the target and a strong X-ray laser signal was generated simultaneously. The hot spot can be clearly resolved in the image as an increase in line emission, particularly strong for the F-like Fe $3 p-2$ s lines. Fig. 1(a) shows a trace of the line focus along the Ne-like $4 d-2 p(4 C)$ line at $12.1 \AA$. The hot spot shows a $30-40 \%$ increase in intensity. A similar trace, Fig.1(c) along the F-like Fe $3-2$ line at 12 . $8 \AA$ shows $\sim 100 \%$ increase in line intensity at the hot spot. This indicates the good sensitivity of these optically thin $4-2$ and $3-2$ lines as an electron temperature diagnostic. Fig. 2 shows a spectral lineout through the hot spot in Fig. 1 (b). A well-resolved spectrum, $\lambda \delta \lambda>1500$, shows Na-like

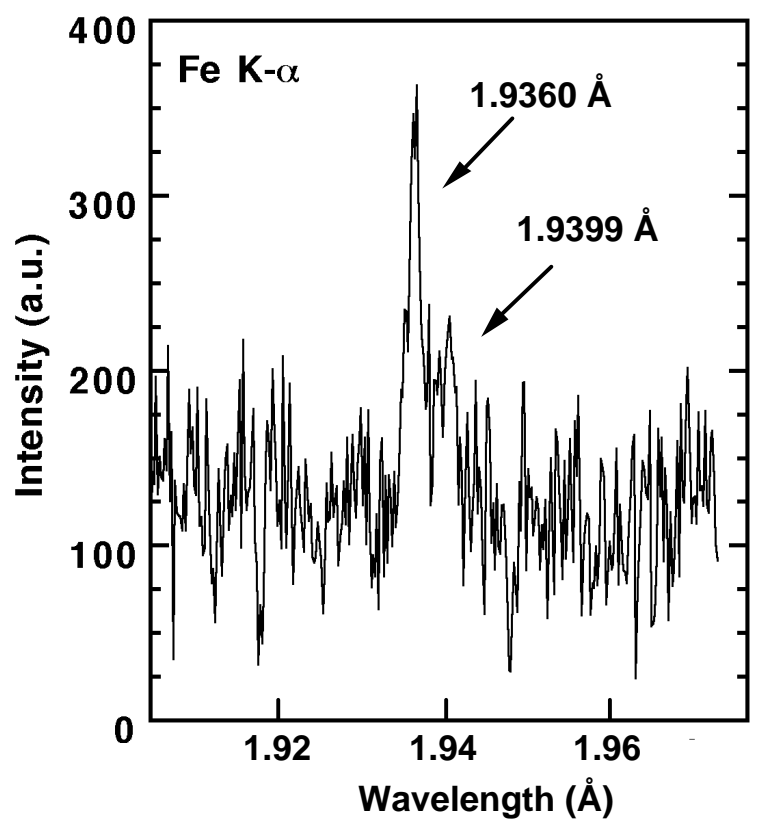

Figure 3 Lineouts through Kodak DEF film showing the Fe K- $\alpha$ doublet spectrum at $1.9360 \AA$ and $1.9399 \AA$ recorded in $8^{\text {th }}$ order of reflection integrated over 40 laser shots. through O-like Fe ion emission. The two strong Ne-like $4 d-2 p$ resonance lines are labeled $4 \mathrm{C}$ and $4 \mathrm{D}$ at 12.12 and $12.26 \AA$. The two groups of lines centered at $\sim 12.4 \AA$ and $12.6 \AA$ are the $\mathrm{Na}$ like satellite lines corresponding to the $4 \mathrm{C}$ and 4D lines. The more intense group of lines above 12.6 $\AA$ come from Fe-like and O-like and are expected to be emitted as the plasma rapidly ionizes after the short pulse. The spectra are being further analyzed and compared with simulations to be reported at a later date.

\section{OBSERVATION OF FE K- $\alpha$ FROM $X$ - RAY LASER TARGETS}

As mentioned in previous experiments for plasma heating using femtosecond or picosecond lasers, a significant fraction of the laser energy can be coupled into superthermal electrons resulting in high energy $10 \mathrm{keV}$ to $\mathrm{MeV}$ bremsstrahlung emission for increasing 
irradiance on target. The nominal peak intensity of the picosecond pulse in this work is $5 \times 10^{14} \mathrm{~W}$ $\mathrm{cm}^{-2}$ which is expected to be high enough, when focused into a preformed plasma with $50-100 \mu \mathrm{m}$ scalelength, to couple some fraction of the laser energy into hot electrons. In this case the presence of hot electrons can be detected by observation of the characteristic K- $\alpha$ radiation from the target material [12]. The FSSR spectrometer with a Mica crystal dispersive element is very convenient for this type of measurement. Indeed, Mica crystals have high reflectivity in many $(n=1,2,3,4,5,7,8$, $11,12,13$ ) higher reflection orders [10]. We could potentially use the same spectrometer to observe the Ne-like Fe XVII resonance lines in the spectral range $\lambda \sim 14-16 \AA$ in $1^{\text {st }}$ order of reflection and simultaneously observe the Fe K- $\alpha_{1,2}(\lambda=1.9360 \AA$ and $\lambda=1.9399 \AA)$ in $8^{\text {th }}$ order of reflection. For better discrimination and selection of Ne-like and K- $\alpha$ lines of Fe we placed a double layer consisting of 2 different $x$-ray films in the FSSR spectrometer film cassette. The top film was Kodak RAR2497 x-ray film, more sensitive to $1 \mathrm{keV}$ x-rays, while the lower film was Kodak Direct Exposure Film (DEF). DEF has better sensitivity to higher energy x-rays in the $4-6 \mathrm{keV}$ range [13]. In this case the $1 \mathrm{keV}$ energy $\mathrm{x}$-rays are fully absorbed on the first film and spectra of $\mathrm{Ne}$-like Fe XVII were recorded there. The $102 \mu \mathrm{m}$ thick polyester $\left(\mathrm{C}_{5} \mathrm{H}_{4} \mathrm{O}_{2}\right)$ base attenuates this longer wavelength radiation. At the same time the RAR film has $50 \times$ less sensitivity to the more energetic $6.4 \mathrm{keV} \mathrm{Fe} \mathrm{K-} \alpha$ x-rays reaching the lower DEF film. The K- $\alpha$ rays are almost fully transmitted (83\%) through the RAR film base and are recorded mainly on the second DEF film. Approximately 40 laser shots were accumulated on the films and a weak but clearly visible Fe K- $\alpha$ signal was observed on the DEF film. Fig. 3 shows a lineout of the spectrum. The two components are resolved and have the expected intensity ratio of 1:0.5. Uncertainty in the $8^{\text {th }}$ order Mica crystal reflectivity prevents an estimate of the absolute $\mathrm{K}-\alpha$ emission strength but substantial hot electrons are being generated with energy in excess of $8-10 \mathrm{keV}$. This demonstrates unequivocally the presence of hot electrons in the plasma plus the effectiveness of the diagnostic technique to simultaneously record but discriminate between sub-kilovolt and multi-kilovolt x-rays.

\section{Acknowledgements}

The authors would like to thank M. Eckart and A. Hazi (LLNL) for continued encouragement and support of this research. The technical contributions from J. Hunter, B. Sellick, J. Ticehurst and A. Ellis (LLNL) are appreciated. This work was performed under the auspices of The U.S. Department of Energy by the University of California Lawrence Livermore National Laboratory under contract No. W-7405-Eng-48.

\section{References}

[1] Yu.V. Afanasiev and V.N. Shlyaptsev, Sov. J. Quant. Electron. 19, 1606 (1989); V.N. Shlyaptsev et al., Proc. SPIE Int. Soc. Opt. Eng. 2012, 111 (1993).

[2] P.V. Nickles et al., Phys. Rev. Lett. 78(14), 2748 (1997).

[3] J. Dunn et al., Phys. Rev. Lett. 80, 2825 (1998).

[4] M.P. Kalachnikov et al., Phys. Rev. A 57, 4778 (1998); P.J. Warwick et al., J. Opt. Soc. Am. B 15(6), 1808 (1998).

[5] M. Nantel, A. Klisnick, G. Jamelot, P. B. Holden, B. Rus, A. Carillon, P. Jaeglé, Ph. Zeitoun, G. Tallents, A. G. MacPhee, C. L. S. Lewis, S. Jacquemot, and L. Bonnet, Phys. Rev. E 54, 2852 (1996).

[6] J.-C. Kieffer, M. Chaker, H. Pépin, M. Nantel, H.A. Baldis, J. Dunn, G.D. Enright and D.M. Villeneuve, Opt. Commun. 84, 208 (1991).

[7] J. Dunn et al., Phys. Rev. Lett. 84, 4834 (2000).

[8] S. J. Moon et al., SPIE Int. Soc. Opt. Eng. 3776, 9 (1999).

[9] T. A. Pikuz, A.Ya. Faenov, S.A. Pikuz, V.M. Romanova, T.A. Shelkovenko. J. X-Ray Sci. Technol. 5, 323 (1995).

[10] A.Ya. Faenov, S.A. Pikuz, A.I. Erko, B.A. Bryunetkin, V.M. Dyakin, G.V. Ivanenkov, A.R. Mingaleev, T.A. Pikuz, V.M. Romanova, T.A. Shelkovenko. Phys. Scr. 50, 333 (1994).

[11] J. Dunn, A.Ya. Faenov, T. A. Pikuz, A.L. Osterheld, et al., in preparation for submission to Rev. Sci. Instrum. (2001). 
[12] J.D. Hares, J.D. Kilkenny, M.H. Key, and J.G. Lunney, Phys. Rev. Lett. 42, 1216 (1979).

[13] B.L. Henke, J.Y. Uejio, G.F. Stone, C.H. Dittmore, and F.G. Fujiwara, J. Opt. Soc. Am. B 3, 1540 (1986). 